\title{
Comparison of the FDA and ASCO/CAP Criteria for HER2 Immunohistochemistry in Upper Urinary Tract Urothelial Carcinoma
}

\author{
Gilhyang Kim ${ }^{1}$. Yul Ri Chung ${ }^{1}$ \\ Bohyun $\mathrm{Kim}^{1} \cdot$ Boram Song ${ }^{1}$ \\ Kyung Chul Moon ${ }^{1,2}$ \\ 'Department of Pathology, \\ 2Kidney Research Institute, Medical Research \\ Center, Seoul National University \\ College of Medicine, Seoul, Korea
}

\begin{abstract}
Background: Human epidermal growth factor receptor 2 (HER2) is one of the known oncogenes in urothelial carcinoma. However, the association between HER2 and the prognosis of upper urinary tract urothelial carcinoma (UUTUC) has not yet been fully clarified. The aim of this study was to evaluate HER2 expression using the United States Food and Drug Administration (FDA) criteria and American Society of Clinical Oncology/College of American Pathologists (ASCO/CAP) criteria and compare their prognostic significance in UUTUC. Methods: HER2 expression was evaluated in 144 cases of UUTUC by immunohistochemistry (IHC) using tissue microarrays. We separately analyzed HER2 expression using the FDA and ASCO/CAP criteria. The $\mathrm{HHC}$ results were categorized into low $(0,1+)$ and high $(2+, 3+)$ groups. Results: Using the FDA criteria, 94 cases were negative, 38 cases were $1+$, nine cases were $2+$, and three cases were $3+$. Using the ASCO/CAP criteria, 94 cases were negative, 34 cases were $1+, 13$ cases were $2+$, and three cases were $3+$. Four cases showing $2+$ according to the ASCO/CAP criteria were reclassified as $1+$ by the FDA criteria. High HER2 expression by both the FDA criteria and ASCO/CAP criteria was significantly associated with International Society of Urological Pathology high grade $(p=.001$ and $p<.001)$. The high HER2 expression group classified with the FDA criteria showed significantly shorter cancer-specific survival $(p=.004)$, but the HER2 high and low expression groups classified with the ASCO/CAP criteria did not show significant differences $(p=.161)$ in cancer-specific survival. Conclusions: HER2 high expression groups were significantly associated with shorter cancer-specific survival, and our study revealed that the FDA criteria are more suitable for determining HER2 expression in UUTUC.
\end{abstract}

Key Words: Upper urinary tract urothelial carcinoma; HER2; FDA criteria; ASCO/CAP criteria
Upper urinary tract urothelial carcinoma (UUTUC), urothelial carcinoma of the renal pelvis and ureter, is a relatively rare malignant tumor, and is known to have a less favorable prognosis because of its late diagnosis compared with urothelial carcinoma of the urinary bladder. ${ }^{1-3}$

Human epidermal growth factor receptor type 2 (HER2) is a transmembrane receptor tyrosine kinase. ${ }^{4}$ HER2 protein overexpression is well known to be associated with poor prognosis in various cancers, and chemotherapy for HER2 has been established for breast cancers, ${ }^{5,6}$ gastric cancers, and gastroesophageal junction carcinomas. $^{7-9}$ In HER2 immunohistochemical staining (IHC), a 3+ HER2 IHC score is regarded as positive, and patients with breast cancer who have a 3+HER2 IHC score are eligible for trastuzumab therapy, but $2+$ HER2 IHC score is regarded as an equivocal result and requires further testing. ${ }^{10,11}$ Many studies have attempted to clarify more accurate guidelines for interpreting HER2 protein expression in various cancers. In breast cancers, the American Society of Clinical Oncology/College of American Pathologists (ASCO/CAP) and United States Food and Drug Administration (FDA) guidelines for HER2 testing, including immunohistochemistry, have been studied and used for years. However, these two guidelines have somewhat different criteria for HER2 IHC. ${ }^{12}$

There have been many reports with respect to HER2 protein overexpression in urothelial carcinomas, and the frequency of HER2 overexpression in urothelial carcinoma is approximately $10 \%{ }^{13-17}$ Some studies showed that HER2 overexpression was a significant prognostic factor in urothelial carcinoma, ${ }^{16}$ and recently, HER2 has been regarded as a new therapeutic target for urothelial carcinomas. ${ }^{14}$ However, a relatively small number of studies have reported HER2 protein expression in UUTUC compared with urothelial carcinoma of the urinary bladder. ${ }^{1-3,16}$

The prognostic significance of HER2 status in UUTUC is still controversial. A few studies showed that HER2 overexpression 
could predict disease progression and disease-related survival in upper urothelial carcinoma, ${ }^{1,3}$ but another study could not show the prognostic significance of HER2 protein expression. ${ }^{2}$ Furthermore, there has been no study comparing the FDA and ASCO/ CAP guidelines in HER2 IHC of UUTUC.

Because the association between HER2 and the prognosis of UUTUC has been controversial, we aimed to compare the prognostic significance of HER2 protein expression using the FDA criteria and the ASCO/CAP guidelines to evaluate which one is more valuable to evaluate the prognosis in UUTUC.

\section{MATERIALS AND METHODS}

\section{Case selection and tissue microarray construction}

A total of 148 cases of UUTUC diagnosed in the Department of Pathology, Seoul National University Hospital, were collected from the computerized database between 1998 and 2005. Hematoxylin and eosin-stained slides were reviewed to determine the pathological diagnosis, tumor grading and pTNM staging compared with the pathology report using the World Health Organization (WHO) classification and American Joint Committee on Cancer (AJCC) staging system. Four cases were excluded because of an inadequate amount of tissue, and eventually, 144 cases were included. The clinical records including age, sex, recurrence, metastasis and survival were collected. We produced tissue microarray (TMA) blocks from formalin-fixed, paraffin-embedded tissue blocks (SuperBioChips Laboratories, Seoul, Korea). Two representative core sections ( $2 \mathrm{~mm}$ in diameter) were taken from the viable tumor area. This study was approved by the Institutional Review Board (IRB) of Seoul National University Hospital.

\section{HER2 immunohistochemistry}

HER2 IHC was performed with the HercepTest kit (Dako, Glostrup, Denmark) according to the manufacturer's protocols. We cut 4- $\mu$ m-thick sections from the TMA block for HER2 IHC. HER2 expression was separately analyzed using FDA and ASCO/CAP 2013 criteria. Using the FDA criteria, the $2+$ positive cases exhibited weak-to-moderate complete membrane staining in $>10 \%$ of tumor cells, ${ }^{18}$ whereas the ASCO/CAP 2013 criteria defined the $2+$ positive cases as circumferential membrane staining that is incomplete and/or weak-to-moderate and present within $>10 \%$ of the invasive tumor cells, or complete and circumferential membrane staining that is intense and present within $\leq 10 \%$ of the tumor cells. ${ }^{12}$ The HER2 IHC results were subcategorized into low $(0,1+)$ and high $(2+, 3+)$ expression groups.

\section{Statistical analysis}

Fisher exact test and Pearson's chi-square test were performed to analyze the correlations between the clinicopathological characteristics and HER2 expression. The cancer-specific survival period was measured from the time of primary surgery to cancerrelated death or the last follow-up. The Kaplan-Meier curves were plotted and the log-rank test was used for the univariate analysis of cancer-specific survival. Cox proportional hazards models was used for the multivariate analysis. In all tests, a twotailed $\mathrm{p}<.05$ was considered statistically significant. SPSS software was used for the statistical analysis (IBM SPSS ver. 21.0, IBM Co., Armonk, NY, USA).

\section{RESULTS}

\section{Clinical and pathological characteristics}

A total of 144 UUTUC cases were included in the present study, of which 108 cases were male and 36 cases were female. The mean age was 66 years old (range, 43 to 87 years). The average tumor size was $4.2 \mathrm{~cm}$ (range, 0.2 to $14.0 \mathrm{~cm}$ ). Lymph node metastasis was observed in nine cases, and distant metastasis was observed in two cases at the time of surgery. Of the 144 cases, 14 were categorized as pTNM stage $0(9.7 \%), 36$ as stage 1 (25.0\%), 23 as stage $2(16.0 \%), 39$ as stage $3(27.1 \%)$, and 32 as stage $4(22.2 \%)$. Sixty-one cases were International Society of Urological Pathology (ISUP) low grade and 83 cases were ISUP high grade. The mean follow-up period was 79 months (range, 2 to 201 months).

\section{HER2 immunohistochemistry}

Using the FDA criteria, 94 cases (65.3\%) were negative, 38 cases $(26.4 \%)$ had a score of $1+$, nine cases $(6.3 \%)$ were $2+$, and three cases (2.1\%) were $3+$ (Fig. 1). Using the ASCO/CAP criteria, 94 cases $(65.3 \%)$ were negative, 34 cases $(23.6 \%)$ were $1+, 13$ cases $(9.0 \%)$ were $2+$, and three cases $(2.1 \%)$ were $3+$. Four cases showing $2+$ IHC result according to the ASCO/CAP 2013 criteria were reclassified as $1+$ by the FDA criteria due to the incomplete membrane staining (Fig. 2).

\section{Correlation between HER2 expression and the clinicopathological characteristics}

The relationship between HER2 expression and the clinicopathological parameters is shown in Table 1. ISUP high grade was associated with high HER2 expression using both the FDA criteria ( $\mathrm{p}=.001)$ and ASCO/CAP 2013 criteria $(\mathrm{p}<.001)$. Other clinical and pathologic characteristics such as sex $(\mathrm{p}>.990$ and $\mathrm{p}=$ 

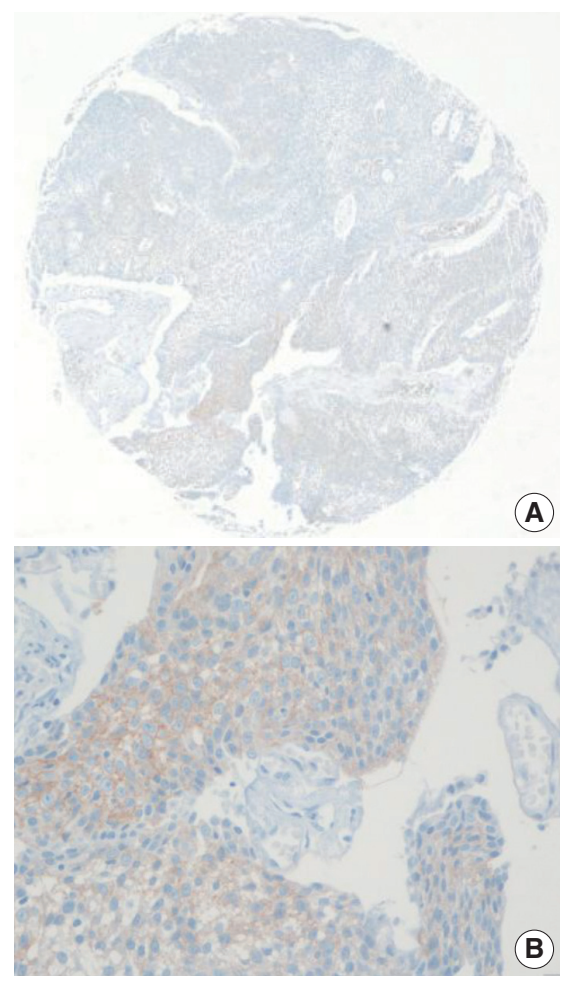
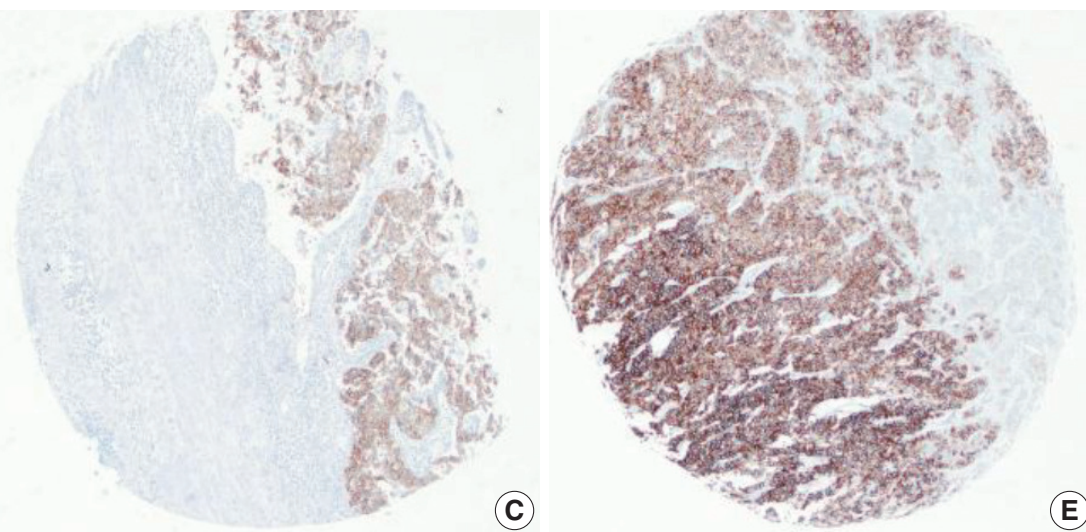

(E)

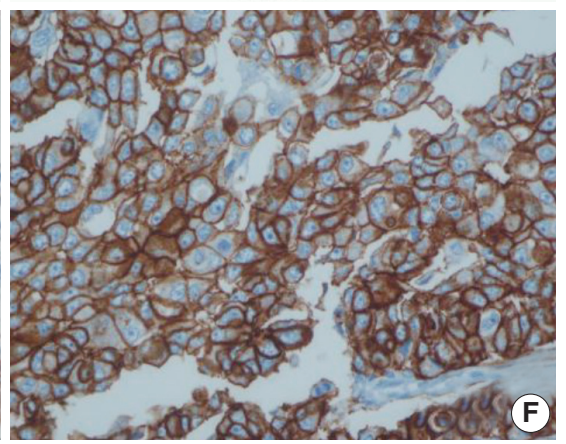

Fig. 1. Representative images of human epidermal growth factor receptor 2 (HER2) expression using the United States Food and Drug Administration criteria. (A, B) 1+ HER2 immunohistochemistry (IHC) positivity, faint membrane staining in more than 10\% of tumor cells. (C, D) 2+ HER2 IHC positivity, moderate complete membrane staining in more than $10 \%$ of tumor cells. (E, F) 3+ HER2 IHC positivity, strong, complete membrane staining in more than $10 \%$ of tumor cells.

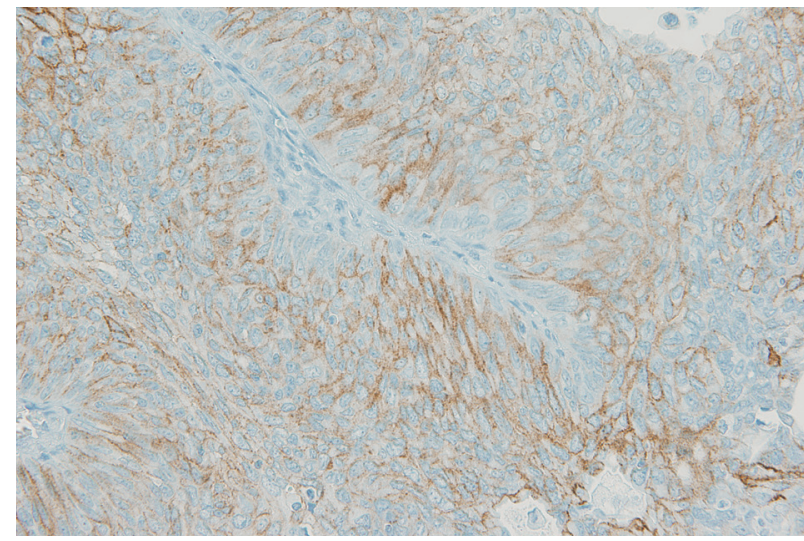

Fig. 2. One of four cases with discrepant scores according to the United States Food and Drug Administration (FDA) and American Society of Clinical Oncology/College of American Pathologists (ASCO/CAP) 2013 criteria. This case is classified as 2+ by the ASCO/CAP guidelines, showed moderate and "incomplete" membrane staining in more than $10 \%$ of tumor cells, and is classified as $1+$ by the FDA guidelines due to the absence of "complete" membrane staining.

.547), age ( $\mathrm{p}=.761$ and $\mathrm{p}=.430)$, size $(\mathrm{p}=.374$ and $\mathrm{p}=.289)$, $\mathrm{T}$ stage $(\mathrm{p}>.990$ and $\mathrm{p}=.604), \mathrm{N}$ stage $(\mathrm{p}=.554$ and $\mathrm{p}>.990), \mathrm{M}$ stage $(\mathrm{p}>.990$ and $\mathrm{p}>.990)$, and $\mathrm{pTNM}$ stage $(\mathrm{p}=.560$ and $\mathrm{p}>$
.990) were not associated with HER2 expression using both the FDA and ASCO/CAP 2013 criteria.

\section{Survival analysis}

The ISUP high grade, pTNM high stage, and HER2 high expression groups, as classified by the FDA criteria, were significantly associated with a shorter cancer-specific survival (Fig. 3AC). However, HER2 high expression group, as classified by the ASCO/CAP 2103 criteria, did not show a significant association with a shorter cancer-specific survival ( $\mathrm{p}=$.161) (Fig. 3D).

In the multivariate Cox regression analysis, pTNM and HER2 expression, as determined by using the FDA criteria, were independent prognostic factors for cancer-specific survival (Table 2).

\section{DISCUSSION}

In this study, we evaluated HER2 protein expression by immunohistochemistry in 144 cases of UUTUC using the following two guidelines: the FDA criteria and ASCO/CAP 2013 criteria. The definitions of IHC $1+$ and $2+$ are different between the two guidelines, and the IHC results from two guidelines showed dif- 
ferent prognostic significance in this study. The numbers of $1+$ and $2+$ positive cases were different, depending on the scoring system. Four cases that scored 2+ using the ASCO/CAP 2013 criteria were reclassified as $1+$ by the FDA criteria. This discrepancy resulted from differences in the definitions of $2+$ and $1+$ positive cases between the two criteria. The FDA criteria classified the $2+$ positive cases as having weak-to-moderate "complete" membrane staining in more than $10 \%$ of tumor cells, whereas the ASCO/CAP 2013 criteria defined the $2+$ positive cases as having circumferential membrane staining that is "incomplete" and/or weak-to-moderate and present within $>10 \%$ of the invasive tumor cells, or complete and circumferential membrane staining that is intense and present within $\leq 10 \%$ of the invasive tumor cells. In previous studies, the rates of $2+13+$ HER 2 IHC in UUTUC ranged from $14 \%$ to $18 \% .^{1-3}$ In this study, the $2+13+$ HER 2 IHC rate was somewhat lower than those of previous studies $(8.3 \%$ FDA criteria and $11.1 \%$ ASCO/CAP 2013 criteria in this study).

The high pTNM stage, high ISUP grade, and high HER2 expression group, as classified by the FDA criteria, showed significantly shorter cancer-specific survival, whereas HER2 expression classified using the ASCO/CAP 2013 criteria did not show a significant association with shorter cancer-specific survival. The multivariate Cox regression analysis indicated that the HER2 IHC results classified by the FDA criteria could be an independent prognostic factor for cancer-specific survival in patients with UUTUC. These results suggest that the FDA guidelines would be more suitable for analyzing HER2 IHC in UUTUC than the ASCO/CAP 2013 guidelines. We think that these results may be important for predicting survival in actual clinical settings.

Some studies have tried to clarify the association between HER2 status and the prognosis of urothelial carcinoma, and the results were diverse. Langner et al. ${ }^{1}$ reported that patients with score 2+ with the FDA guidelines had a significant correlation with metastasis-free survival. Vershasselt-Crinquette et al. ${ }^{2}$ used the ASCO/CAP criteria and their result showed that HER2 overexpression was not correlated with specific survival or recurrence. Sasaki et al. ${ }^{13}$ revealed that HER2 positive patients showed a significant association with shorter time to recurrence using their own criteria according to the ASCO/CAP criteria and the results of the Trastuzumab for Gastric Cancer (ToGA)

Table 1. Association between the clinicopathological characteristics and HER2 expression

\begin{tabular}{|c|c|c|c|c|c|c|}
\hline \multirow{3}{*}{ Characteristic } & \multicolumn{6}{|c|}{ HER2 score } \\
\hline & \multicolumn{3}{|c|}{ FDA criteria } & \multicolumn{3}{|c|}{ ASCO/CAP 2013 criteria } \\
\hline & 0 and $1+$ & $2+$ and $3+$ & $p$-value & 0 and $1+$ & $2+$ and $3+$ & $p$-value \\
\hline Sex & & & $>.990$ & & & .547 \\
\hline Male & 99 & 9 & & 97 & 11 & \\
\hline Female & 33 & 3 & & 31 & 5 & \\
\hline Age (yr) & & & .761 & & & .430 \\
\hline$<66$ & 56 & 4 & & 55 & 5 & \\
\hline$\geq 66$ & 76 & 8 & & 73 & 11 & \\
\hline Size (cm) & & & .374 & & & .289 \\
\hline$<4.2$ & 80 & 9 & & 77 & 12 & \\
\hline$\geq 4.2$ & 52 & 3 & & 51 & 4 & \\
\hline T stage & & & $>.990$ & & & .604 \\
\hline $\mathrm{Ta}$, Tis and $\mathrm{T} 1, \mathrm{~T} 2$ & 73 & 7 & & 70 & 10 & \\
\hline T3 and T4 & 59 & 5 & & 58 & 6 & \\
\hline N stage & & & .554 & & & $>.990$ \\
\hline NO & 124 & 11 & & 120 & 15 & \\
\hline N1, N2 and N3 & 8 & 1 & & 8 & 1 & \\
\hline M stage & & & $>.990$ & & & $>.990$ \\
\hline MO & 130 & 12 & & 126 & 16 & \\
\hline M1 & 2 & 0 & & 2 & 0 & \\
\hline pTNM stage & & & .560 & & & $>.990$ \\
\hline Oa, Ois, I and II & 68 & 5 & & 65 & 8 & \\
\hline III and IV & 64 & 7 & & 63 & 8 & \\
\hline ISUP & & & .001 & & & $<.001$ \\
\hline Low & 61 & 0 & & 61 & 0 & \\
\hline High & 71 & 12 & & 67 & 16 & \\
\hline
\end{tabular}

HER2, human epidermal growth factor receptor 2; FDA, United States Food and Drug Administration; ASCO/CAP, American Society of Clinical Oncology/College of American Pathologists; ISUP, International Society of Urological Pathology. 
trial. Tsai et al. ${ }^{3}$ also used their own scoring system and showed the result that HER2 expression predicted for disease progression and disease-related survival. We thought that the diverse results
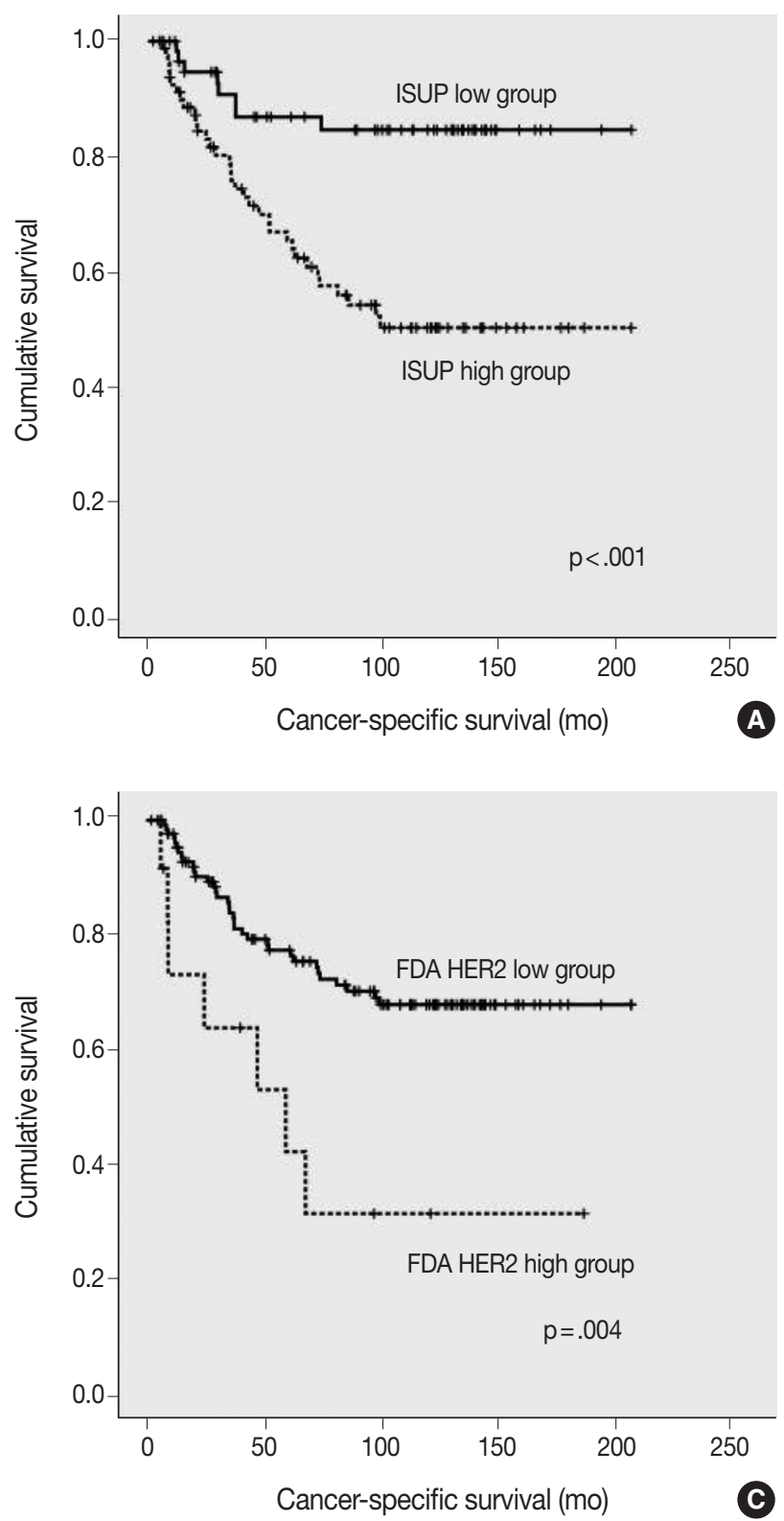

could be caused by the different scoring system for HER2 IHC Furthermore, there has been no study that compared the FDA and ASCO/CAP guidelines for interpretation of HER2 IHC in
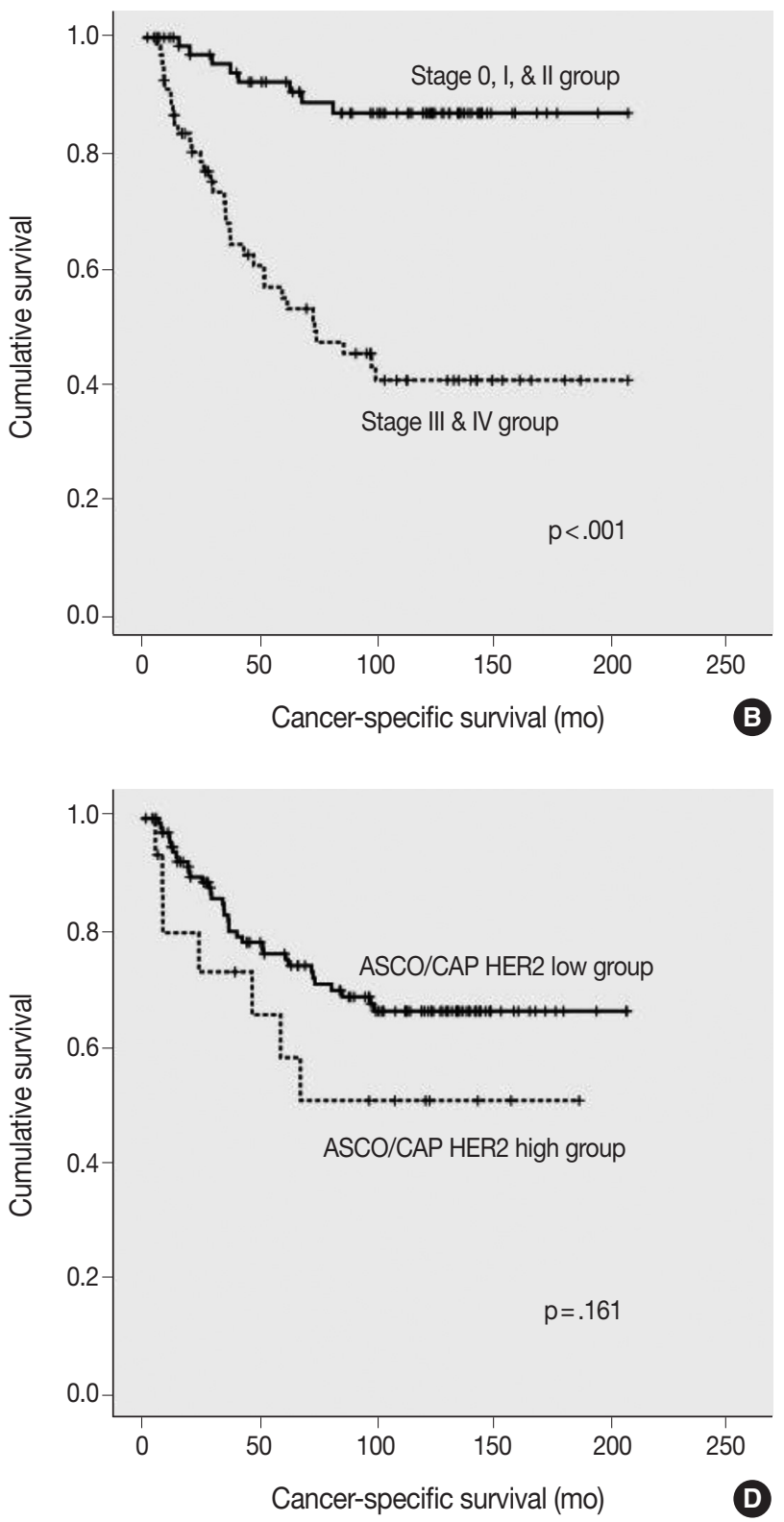

Fig. 3. Kaplan-Meier survival curves for cancer-specific survival. (A) Cancer-specific survival according to the International Society of Urological Pathology (ISUP) grade. (B) Cancer-specific survival according to the pTNM stage. (C) Cancer-specific survival according to human epidermal growth factor receptor 2 (HER2) expression by the United States Food and Drug Administration (FDA) criteria. (D) Cancer-specific survival according to HER2 expression by the American Society of Clinical Oncology/College of American Pathologists (ASCO/CAP) criteria.

Table 2. Multivariate Cox regression analysis of cancer-specific survival

\begin{tabular}{lllr}
\hline & & Hazard ratio (95\% Cl) & p-value \\
\hline ISUP grade & High vs Low & $1.710(0.739-3.958)$ & .210 \\
pTNM stage & III, IV vs 0, I, II & $5.153(2.267-11.715)$ & $<.001$ \\
HER2 expression (FDA) & High vs Low & $2.478(1.073-5.724)$ & .034 \\
\hline
\end{tabular}

$\mathrm{Cl}$, confidence interval; ISUP, International Society of Urological Pathology; HER2, human epidermal growth factor receptor 2; FDA, United States Food and Drug Administration. 
UUTUC. In this study, we compared the FDA and ASCO/CAP guidelines for HER2 IHC in UUTUC, and found that the prognostic significance of HER2 IHC was different between the two guidelines.

We did not confirm the HER2 gene amplification status by fluorescence in situ hybridization analysis, and this is the limitation of the present study. There have been some studies reporting good correlation between HER2 protein overexpression and gene amplification in UUTUC., ${ }^{2,13}$

In conclusion, our study indicated that the HER2 IHC result has significant prognostic implications and the FDA criteria are more suitable for interpreting the HER2 IHC results in UUTUC than the ASCO/CAP criteria in UUTUC.

\section{Conflicts of Interest}

No potential conflict of interest relevant to this article was reported.

\section{Acknowledgments}

This work was supported by a grant 04-2016-0460 from the Seoul National University Hospital Research Fund.

\section{REFERENCES}

1. Langner C, Gross C, Rehak P, Ratschek M, Ruschoff J, Zigeuner R. HER2 protein overexpression and gene amplification in upper urinary tract transitional cell carcinoma: systematic analysis applying tissue microarray technique. Urology 2005; 65: 176-80.

2. Vershasselt-Crinquette M, Colin P, Ouzzane A, et al. Assessment of human epidermal growth factor receptor 2 status in urothelial carcinoma of the upper urinary tract: a study using dual-color in situ hybridization and immunohistochemistry. Appl Immunohistochem Mol Morphol 2012; 20: 363-6.

3. Tsai YS, Tzai TS, Chow NH, Wu CL. Frequency and clinicopathologic correlates of ErbB1, ErbB2, and ErbB3 immunoreactivity in urothelial tumors of upper urinary tract. Urology 2005; 66: 1197-202.

4. Olayioye MA, Neve RM, Lane HA, Hynes NE. The ErbB signaling network: receptor heterodimerization in development and cancer. EMBO J 2000; 19: 3159-67.

5. Tinoco G, Warsch S, Gluck S, Avancha K, Montero AJ. Treating breast cancer in the 21st century: emerging biological therapies. J Cancer 2013; 4: 117-32.

6. Hammond ME, Hayes DF, Dowsett M, et al. American Society of Clinical Oncology/College Of American Pathologists guideline recommendations for immunohistochemical testing of estrogen and progesterone receptors in breast cancer. J Clin Oncol 2010; 28: 2784-95.

7. Smyth EC, Cunningham D. Targeted therapy for gastric cancer. Curr Treat Options Oncol 2012; 13: 377-89.

8. Bang YJ. Advances in the management of HER2-positive advanced gastric and gastroesophageal junction cancer. J Clin Gastroenterol 2012; 46: 637-48

9. Kunz PL, Mojtahed A, Fisher GA, et al. HER2 expression in gastric and gastroesophageal junction adenocarcinoma in a US population: clinicopathologic analysis with proposed approach to HER2 assessment. Appl Immunohistochem Mol Morphol 2012; 20: 13-24.

10. Chibon F, de Mascarel I, Sierankowski G, et al. Prediction of HER2 gene status in Her2 2+ invasive breast cancer: a study of 108 cases comparing ASCO/CAP and FDA recommendations. Mod Pathol 2009; 22: 403-9.

11. Slamon DJ, Leyland-Jones B, Shak S, et al. Use of chemotherapy plus a monoclonal antibody against HER2 for metastatic breast cancer that overexpresses HER2. N Engl J Med 2001; 344: 783-92.

12. Wolff AC, Hammond ME, Hicks DG, et al. Recommendations for human epidermal growth factor receptor 2 testing in breast cancer: American Society of Clinical Oncology/College of American Pathologists clinical practice guideline update. J Clin Oncol 2013; 31: 3997-4013

13. Sasaki Y, Sasaki T, Kawai T, et al. HER2 protein overexpression and gene amplification in upper urinary tract urothelial carcinoma-an analysis of 171 patients. Int J Clin Exp Pathol 2014; 7: 699-708.

14. Lae M, Couturier J, Oudard S, Radvanyi F, Beuzeboc P, Vieillefond A. Assessing HER2 gene amplification as a potential target for therapy in invasive urothelial bladder cancer with a standardized methodology: results in 1005 patients. Ann Oncol 2010; 21: 815-9.

15. Hansel DE, Swain E, Dreicer R, Tubbs RR. HER2 overexpression and amplification in urothelial carcinoma of the bladder is associated with MYC coamplification in a subset of cases. Am J Clin Pathol 2008; 130: 274-81.

16. Krüger S, Weitsch $G$, Büttner $H$, et al. HER2 overexpression in muscle-invasive urothelial carcinoma of the bladder: prognostic implications. Int J Cancer 2002; 102: 514-8.

17. Ruschoff J, Hanna W, Bilous M, et al. HER2 testing in gastric cancer: a practical approach. Mod Pathol 2012; 25: 637-50.

18. Buza N, English DP, Santin AD, Hui P. Toward standard HER2 testing of endometrial serous carcinoma: 4-year experience at a large academic center and recommendations for clinical practice. Mod Pathol 2013; 26: 1605-12. 